\title{
Bronchial Artery Embolization - An Unusual Cause of Paraplegia and Review of Literature
}

\author{
Authors: Lekhra OP ${ }^{1}$, Dosi $\mathrm{R}^{2}$ \\ ${ }^{1}$. Associate Professor Department of Neurology \\ 2. Assistant Professor Department of T.B. and Chest Diseases \\ Sri Aurobindo Medical College \& PG Institute, Indore, INDIA
}

\begin{abstract}
Bronchial artery embolization (BAE) is a well-established minimally invasive procedure in the management of moderate to massive and recurrent hemoptysis. The bronchial circulation aneurysms and dilatations are commonly responsible for the hemoptysis and have an easy approach through the descending aorta via the femoral or subclavian route by angiography. There are very few common complications associated with the procedure are recurrence of hemoptysis, chest pain, cough, dysphagia .Paraplegia secondary to embolization of the anterior spinal artery is a very rare but serious complication. Proper identification of the culprit bronchial vessel and pre-embolization study of its anastomosis are useful measures to minimize the chances of this dreaded complication.
\end{abstract}

Key Words: Bronchial artery embolization, Recurrent Hemoptysis, Paraplegia, Gel foam

\section{Introduction}

The pulmonary circulation comprises of the pulmonary \& bronchial group of vessels. The bronchial arteries arise from the systemic high pressure descending aorta circulation whereas the pulmonary artery arises from the right ventricle where it brings deoxygenated blood to the lungs for oxygenation. The dilatation of the pulmonary circulation at different sites or formation of bronchopulmonary anastamosis result in the formation of Rasmussen's aneurysm's which in turn may result in hemoptysis. Hemoptysis is a distressing and potentially fatal complication of various pulmonary disorders of which tuberculosis is an important and common etiology. The medical management of hemoptysis offers limited benefit and hence multiple interventional procedures both minimal and radically interventional have been evolved to treat the condition. Bronchial artery embolization (BAE) is one such minimal technique which is extremely effective with a low chance of complications and failure.

\section{Case Report}

A 56 year old male store keeper by occupation, resident of an urban area presented to the hospital with the complaints of recurrent blood streaked expectoration for the past five months. The patient was admitted and started on medical management in the form of styptics, antibiotics, anti-inflammatory drugs and hematinics. His blood investigations revealed : $\mathrm{Hb} 11.5 \mathrm{mg} \%$,WBC-7100.ESR-28mm/1 Hr,PCV-47.2,sputum AFB-negative at two occasions, PTT test-33 seconds control-30 seconds, APTT test 16 seconds and control 13 seconds, Blood urea-22,serum creatinine-1.29, alkaline phosphatase-81,SGOT-38,SGPT-60,,HIV \& HbsAg-negative,lipid profile-normal, Serum homocysteine-9.62micromoles/L, urine examination was unremarkable, Spirometry revealed around 50\% restrictive findings and bronchoalveolar lavage examination negative for acid fast bacilli .The patient attained partial control of hemoptysis and was discharged on anti tubercular treatment. Six months after discharge, the patient completed a regular course of anti tubercular treatment but was persistently symptomatic with hemoptysis. He presented with an episode of massive hemoptysis for three days and was admitted to the chest ward and underwent evaluation with a contrast CT scan. The CT scan revealed a right upper lobe fibrocavitory disease with a possibility of a dilated Rasmussen's aneurysm. The fibreoptic bronchoscopy of the patient was done and a normal bronchial tree was visualized .Retained hemorrhagic and purulent secretions were visualized which on subsequent analysis showed methicillin sensitive streptococcus pneumonia for which appropriate treatment was given. The patient had other co-morbidities in the form of hypertension for the past ten years for which he was regular antihypertensive. In view of the localized pathology and potentially fatal nature of his complication, bronchial artery embolization was offered to the patient. His pulmonary angiogram study was performed with iodine based contrast and a culprit vessel was identified in the right upper lobe branch of the right bronchial artery which was eventually embolized with gel foam. Pre and post embolization the patency of the other systemic and spinal arteries was performed with lateral imaging using fluoroscopic vision.

Few hours post procedure he started to develop tingling and numbness in both lower limbs with severe pain for which analgesics were administered and hemodynamic assessment was done to rule out any hypotension. $\mathrm{He}$ 
was examined neurologically to rule out any weakness of the lower limbs but power was grade $5 / 5$ at that time. Over the next twenty four hours he developed severe pain in both his lower limbs which evolved further into full fledged into paraplegia. On examination patient was conscious and oriented, BP 130/80 Hg right upper limb supine, PR 86/min, RR 20/min, higher function, cranial nerves and upper limb examination were unremarkable. Lower limb were hypotonic, power 0/5, DTR absent in lower limbs, 50\% pin prick sensory loss below T10 level, posterior column sensation were intact and plantars were extensor bilaterally. A provisional diagnosis of acute paraplegia with a differential diagnosis of epidural hematoma, epidural abscess and spinal cord infarction was made .MRI was done which ruled out epidural causes and a final diagnosis of anterior spinal infarct was confirmed (Fig 1a, b, c).Patient was managed symptomatically and all the rehabilitation modalities for, back, bladder, bowel and limbs were given. On follow up respiratory consultation it was found that the patient's chest symptoms improved and had no episode of hemoptysis thereafter. Neurological examination at follow up after three months revealed improvement in motor power (Grade 3/5) .He could walk few steps with support. Patient's respiratory symptoms were also much better.

\section{Discussion:}

The evaluation of an individual with an acute paraplegia is best performed with a differential diagnosis in mind. The differential diagnosis of paraplegia includes spinal cord injury, tumor, infection, demyelination, vascular insult and abdominal aortic occlusion/dissection. An immediate total loss of function is almost always vascular. The blood supply to spinal cord is supplied by one anterior spinal artery and two posterior spinal arteries. As they come down the spinal cord, they are fed by segmental arteries that arise from the aorta which are more for posterior than anterior spinal artery. This is the reason for anterior spinal artery syndrome being more common than the posterior spinal artery syndrome. Most radicular arteries arise from the left extra spinal vessels. Very often, a single, large radicular artery is found. The radiculo-medullary artery (artery of Adamkiewicz) constitutes the largest and most important radicular artery. It enters the spinal canal in variable segments; most commonly from T6 to L1.There is variability in the number and size of the supplying arteriesthat is, both longitudinal arteries and the anterior spinal artery. The variability in the blood supply makes it difficult to identify and spare the specific blood vessels as they arise from the aorta1. Most commonly this creates a difficulty in identification of the vessel which can result in spinal cord ischemia. The blood supply to spinal cord may be involved by numerous mechanisms. 2 these include the inadvertent embolization of the artery of Adamkiewicz or abnormal embolization of the gel foam. 3, 4 Stagnation of blood within the vessels supplying the spinal cord can also occur as a result of prolonged periods of hypotension. Physiological factors can add to the risk including atherosclerosis, polycythemia, and anatomic changes of the spine (kyphoscoliosis), vascular malformations and the hypercoagulability associated with malignancy. An epidural hematoma can also cause extrinsic compression causing paraplegia ${ }^{5}$. Paraplegia resulting from inadequate blood supply through the anterior spinal artery produces a clinical picture known as anterior spinal artery syndrome. This is characterized by loss of motor function with intact or, more commonly, impaired sensory function below the level of injury

Bronchial artery embolization (BAE) is a well-established procedure in the management of massive and recurrent hemoptysis. Embolization in this territory is most commonly performed with particles such as gel foam, PVA or more recently tris-acryl gelatin microspheres ${ }^{6}$. The use of thrombin as an embolic agent has also been recently described ${ }^{7}$.A thorough knowledge of normal and variant bronchial arterial anatomy as well as collateral supply is necessary prior to embarking on any embolization procedure ${ }^{8,9}$. The most dreaded and serious complication of this procedure is spinal cord ischemia secondary to inadvertent embolization of the anterior or posterior spinal arteries via radiculo-medullary arteries, which may arise from the bronchial arteries. This complication has been well described to occur in 1.4 to $6.5 \%$ of BAE according to different published series $^{10,11}$.The use of a micro catheter may be indicated in this situation so that BAE may be performed from a more distal position. Also one must be aware of the presence of any bronchopulmonary anastomosis to either the pulmonary arteries or veins. It has been seen that average size of anastomotic vessels in these circumstances is over $300 \mu \mathrm{m}^{12}$. Hence it is recommended that any particle employed as embolic agent should be larger than this ${ }^{13}$. Moreover, the use of smaller particles will also result in an overly distal occlusion that may impede the normal blood supply to other anatomical structures, such as the esophagus leading to necrosis. That is the reason why liquid embolic agents are no longer preferred ${ }^{13}$ In fact, dysphagia has been described to develop following $\mathrm{BAE}$ in up to $18 \%$ of the cases ${ }^{10,14 . \text { and }}$ the occurrence of a bronchoesophageal fistula has also been reported $^{15}$.Equally, aortic and bronchial necrosis, although rare, may also occur ${ }^{16}$. However, the most commonly reported complication following BAE is chest pain, which has been described to occur in 24 to $91 \%$ of BAE procedures ${ }^{10}$.

In our case the procedure was uneventful with no documented hypotensive episode. The patient recovered in an intensive care unit and required no inotropic support as he was maintaining a mean arterial pressure $>86 \mathrm{mmHg}$. In this patient an epidural hematoma or abscess was among the differential diagnoses as a cause for the paraplegia; however, this was excluded by means of an MRI scan. Super selection of the specific 
artery done, hence no chance of inadvertent ligation of the vessel. Reflux of the gel foam into anterior spinal artery could be the one explanation for the acute paraplegia. Mechanism of gel foam is it temporarily occludes vessels for four-five weeks ${ }^{17}$. Gel foam is composed of water-insoluble gelatin, so the particles travel distally and occlude smaller capillaries. It works by absorbing liquid and plugging the vessel. It has well reported that the eembolic material may reflux and enter other arteries if the injection is performed too rapidly ${ }^{17}$. Once the artery is partially occluded and the blood flow is reduced, further embolization is hazardous because particles tend to reflux out of the vessel. Reflux may also occur as the catheter is withdrawn. Patient gradually showed improvement with symptomatic drugs, antiplatelets, and physiotherapy and rehabilitation manoeuvres. It is therefore important to identify preoperatively patients who are at increased risk of compromised blood flow to the spinal cord, such as elderly patients with known risk factors for atherosclerosis, to implement preventive measures such as bronchial artery embolization. These measures may include preoperative echocardiography, reduced aortic manipulation and thromboprophylaxis both pre/post- operatively. However, the avoidance of hypoxia and hypotension at any stage of the procedure must be paramount.

As a conclusion, BAE is a relatively safe procedure, and most complications related to the procedure are minor. We believe the use of micro catheters for super selective catheterization and embolization may minimize serious complications related to spinal cord injury.
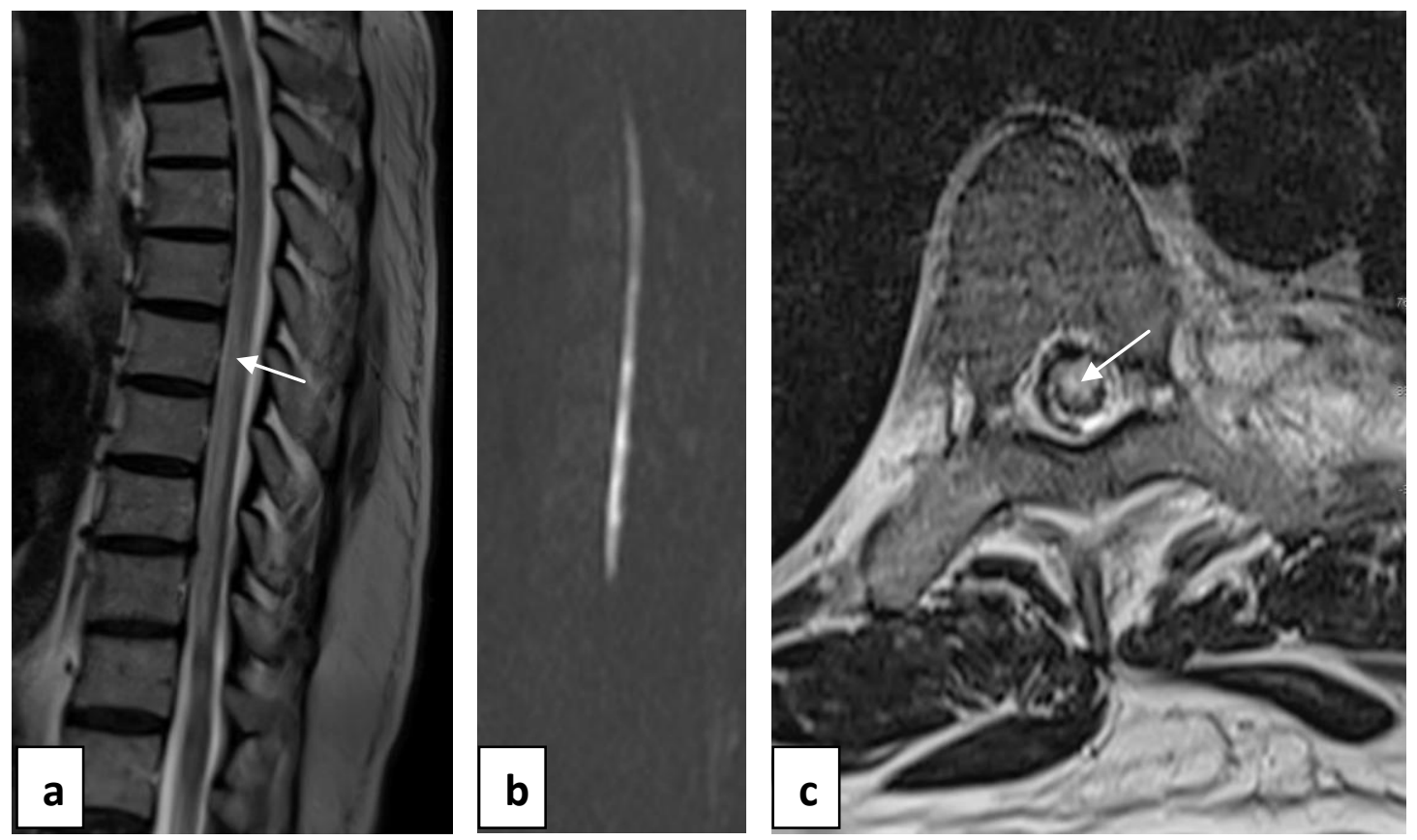

Fig 1a MRI T2W sagittal image showing hyper intense signal (arrow) within the cord extending over dorsal segments (D6-D10).

Fig1b MRI diffusion sagittal image showing only the infarcted anterior part of the spinal cord by the hyper intense signals.

Fig 1c. MRI diffusion axial scans through the same lower dorsal cord level reveal a central intense signal (arrow) sparing the posterior part of the cord. The cord itself is of normal size.

\section{References:}

[1]. Shahi N,Asanta-Siaw J and Marzouk JFK. Paraplegia following oesophagectomy BMJ Case Rep. 2010; 2010: bcr09.2009.2270.

[2]. Cheshire WP, Santos CC, Massey EW, et al. Spinal cord infarction: etiology and outcome. Neurology1996; 47: 321-30. [PubMed]

[3]. Kane RE. Neurologic deficit following epidural and spinal anesthesia. Anesth Analg 1981; 60: 150-614.

[4]. SkounJS,WainapelSF, WillockMM. Paraplegia following epidural anesthesia. Acta Neurol Scand1985;72:437-43. .[PubMed]

[5]. Urquhuat-Hay D. Paraplegia following epidural analgesia. Anesthesiology 1969; 24: 461-70. .[PubMed]

[6]. Fernando HC,Stein M,Benfield JR,Link DP.Role of bronchial artery embolization in the management of hemoptysis.Arch Surg.1998;133:862-866

[7]. Vrachliolitis T,Sheiman RG.Treatment of massive hemoptysis with intrarterial thrombin injection of a bronchial artery.AJR Am J Roentgenol.2002;179:113-114

[8]. McPherson S, Routh W D, Nath H, Keller F S. Anomalous origin of bronchial arteries: potential pitfall of embolotherapy for hemoptysis. J Vasc Interv Radiol. 1990;1:86-88. [PubMed]

[9]. Sancho C, Escalante E, Dominguez J, et al. Embolization of bronchial arteries of anomalous origin. Cardiovasc Intervent Radiol. 1998;21:300-304. [PubMed]

[10]. Ramakantan R, Bandekar V G, Gandhi M S, Aulakh B G, Deshmukh H L. Massive hemoptysis due to pulmonary tuberculosis: control with bronchial artery embolization. Radiology.1996;200:691-694. [PubMed] 
[11]. Wong M L, Szkup P, Hopley M J. Percutaneous embolotherapy for life-threatening hemoptysis.Chest. 2002;121:95-102. [ubMed]

[12]. Pump K K. Distribution of bronchial arteries in the human lung. Chest. 1972;62:447-451.[PubMed]

[13]. Yoon W, Kim J K, Kim Y H, Chung T W, Kang H K. Bronchial and nonbronchial systemic artery embolization for life-threatening hemoptysis: a comprehensive review. Radiographics.2002;22:1395-1409. [PubMed]

[14]. Tonkin I L, Hanissian A S, Boulden T F, et al. Bronchial arteriography and embolotherapy for hemoptysis in patients with cystic fibrosis. Cardiovasc Intervent Radiol. 1991;14:241-246.[PubMed]

[15]. Munk P L, Morris D C, Nelems B. Left main bronchial-esophageal fistula: a complication of bronchial artery embolization. Cardiovasc Intervent Radiol. 1990;13:95-97. [PubMed]

[16]. Girard P, Baldeyrou P, Lemoine G, Grunewald D. Left main-stem bronchial stenosis complicating bronchial artery embolization. Chest. 1990;97:1246-1248.

[17]. Michael L,Charles ER,Funaki B. Embolization Agents-Which One Should Be Used When? Part 1: Large-Vessel Embolization.Semin Intervent Radiol. 2009;(4): 352-357

[18]. Tanaka N, Yamakado K, Murashima S, et al. Superselective bronchial artery embolization for hemoptysis with a coaxial microcatheter system. J Vasc Interv Radiol. 1997;8:65-70. [PubMed] 